\title{
Developmental Combination of Fluoxetine and Buspirone and Prenatal Stress Effects on Pain-Related Behavior of Prepubertal Female Offspring
}

\section{Butkevich IP* and Mikhailenko VA}

Institute of Physiology, Russian Academy of Sciences, St. Petersburg, Russia

*Corresponding author: Butkevich IP, Institute of Physiology, Russian Academy of Sciences, St. Petersburg, Russia

Received: April 09, 2018; Accepted: April 18, 2018;

Published: April 25, 2018

\section{Keywords}

Combination of Fluoxetine and Buspirone; Pregnancy; Prenatal Stress; Development; Female Offspring; Pain; Rat

\section{Short Communication}

A systematic study was made to determine the effects of developmental exposure to a combination of selective serotonin (5-HT) reuptake inhibitor fluoxetine and 5-HT1A receptor partial agonist buspirone on pain sensitivity to thermal stimulus and on the inflammatory pain response in female rat offspring during the prepubertal period of development. Pregnant rat dams from the second week of pregnancy were exposed to daily injection of fluoxetine, or buspirone, or their combination, or saline, and during the last week of pregnancy, all the rats were exposed to restraint stress (prenatal stress) twice a day during an hour. Rat dams not exposed to any effects during pregnancy were also included in the experiments (intact rats). In 25-day-old offspring, pain sensitivity in the hot plate test and then pain-related behavior in response to inflammatory agent in the formalin test $(2.5 \%, 1.0 \mu \mathrm{l}$ formalin into the pad of the left hind paw) were investigated. Prenatal stress decreased the latent period of response (increased pain sensitivity) to thermal stimulus as compared with this index in intact rats. Developmental drugs exposure to prenatally stressed female offspring attenuated pain sensitivity. The influence of developmental exposure to the combination of fluoxetine and buspirone on pain sensitivity in the offspring as compared to the similar influence of fluoxetine alone was more stronger $(\mathrm{p}<0.01)$.

Prenatal stress increased licking duration during the first acute $(\mathrm{p}<0.001)$ and the second tonic $(\mathrm{p}<0.01)$ phases in the formalin test as compared with these indices in prenatally not stressed rats. Developmental exposure to fluoxetine alone failed to change in the offspring licking duration during the first acute phase, whereas similar exposure to buspirone and combination of the drugs normalized pain-related behavior in the offspring and revealed differences with fluoxetine $(\mathrm{p}<0.01)$. Developmental exposure to each from the drugs to prenatally stressed females normalized licking duration during the second tonic phase. Thus, prenatal stress strengthens pain-related behaviors in response to both of thermal and inflammatory stimuli in the female rats, developmental exposure to anxiolytic buspirone, antidepressant fluoxetine (except for the acute phase in the formalin test) or their combination to prenatally stressed offspring approves the pain-related indices under study. Differences in analgesic effects of the drugs were found between fluoxetine and the combination of fluoxetine and buspirone in both nociceptive tests. This study was supported by the Russian Foundation for Basic Research (grant no. 17-04-00214a).
Austin J Anesthesia and Analgesia - Volume 6 Issue 1 - 2018 ISSN : 2381-893X | www.austinpublishing group.com Butkevich et al. () All rights are reserved
Citation: Butkevich IP and Mikhailenko VA. Developmental Combination of Fluoxetine and Buspirone and Prenatal Stress Effects on Pain-Related Behavior of Prepubertal Female Offspring. Austin J Anesthesia and Analgesia. 2018; 6(1): 1068. 